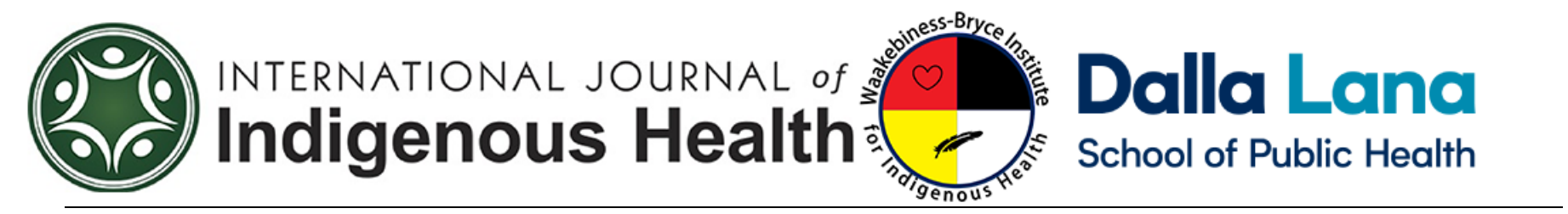

Volume 14

Issue 2. Growing Roots of Indigenous Wellbeing

Full Issue DOI: 10.32799/ijih.v14i2.32958

Article 7

October 2019

\title{
Exploring the health and well-being of children and youth in Winneway, Québec
}

\author{
Alison Kutcher, \\ Ingram School of Nursing, McGill University \\ alison.kutcher@mail.mcgill.ca

\section{Priscilla Pichette} \\ Long Point First Nation Health and Wellness Centre \\ priscillapolson@hotmail.com

\section{Mary Ellen Macdonald,} \\ Division of Oral Health and Society, Faculty of Dentistry, McGill University \\ mary.macdonald@mcgill.ca
}

Franco A. Carnevale

Ingram School of Nursing, McGill University

franco.carnevale@mcgill.ca

\section{Follow the International Journal of Indigenous Health at:}

https://jps.library.utoronto.ca/index.php/ijih/index

\section{Recommended Citation}

Kutcher, A., Pichette, P., Macdonald, ME., \& Carnevale, FA. (2019). Exploring the health and wellbeing of children and youth in Winneway, Québec. International Journal of Indigenous Health, 14(2), 115- 132. DOI 10.32799/ijih.v14i2.31910 


\title{
Exploring the health and well-being of children and youth in Winneway, Québec
}

\begin{abstract}
Health inequalities of Indigenous children and youth in Canada are well documented. Recently, the perspectives of children and youth are being recognized as valuable. However, there is a paucity of literature that seek children and youth's perspective regarding their health and well-being. The purpose of this study was to understand how children and youth in Winneway, QC view health and well-being and to identify their main health and well-being concerns. A focused ethnographic study with Indigenous decolonizing framework was used with data primarily collected through interviews of fifteen participants aged 6 to 17. Children and youth in Winneway view their health and well-being as multidimensional and view themselves as decision-makers in their health and well-being. Their main health and well-being concerns include poor eating choices, difficulty expressing emotional and mental concerns, how children and youth treat others, and youth participation in unhealthy behaviours. These findings reveal the valuable perspectives that Indigenous children and youth can have regarding their health and well-being. They also suggest that future health and well-being interventions targeting Indigenous children and youth seek out and respect the knowledge and perspectives that children and youth have of their health and well-being.
\end{abstract}

\section{Keywords}

Indigenous, Community health, Child health, Youth participation, Well-being, Focused ethnography

\section{Creative Commons License (a) $\Theta \Theta \Theta$}

This work is licensed under a Creative Commons Attribution-Noncommercial-No Derivative Works 4.0 License. 


\section{Introduction}

Poor health disproportionately affects First Nations, Métis, and Inuit peoples in Canada (Government of Canada, 2004). Recent aims at reducing these inequalities and improving Indigenous health and well-being have shown some success in longer life expectancy; however, Indigenous communities still face many challenges today. Recommendations from the Truth and Reconciliation Commission (TRC) (2015) recognize these challenges and call to action the importance of working with Indigenous peoples and communities in a culturally-attuned and respectful manner to address their health and well-being goals and concerns. Indigenous children and youth are a unique population, growing at a faster rate compared to non-Indigenous children and youth in Canada (Government of Canada, 2016). Health inequalities among Canadian and Quebec Indigenous children and youth are reflected by health disparities, including higher rates of obesity, diabetes, infectious diseases, poor oral hygiene, depression, and suicide, compared to their non-Indigenous counterparts (Boksa, Joober, \& Kirmayer, 2015; Crawford A, 2015; Gracey M \& King M, 2009; King, Smith, \& Gracey, 2009, p. 2; Kooiman et al., 2012; Rice et al., 2016). Despite these known health risks, there is a paucity of literature exploring the health and well-being concerns from the perspectives of Indigenous children and youth.

Children and youth are an integral part of their community. Indigenous communities value children and youth and look to them as a source of pride (Ball, 2004). They believe that raising children and youth with a strong sense of cultural identity is key to improve their well-being (Ball, 2004; Public Health Agency of Canada, 2008). Much research has sought the perspective of children and youth through adults, guardians, and healthcare professionals (HCPs), excluding the direct perspectives of children and youth. This view is being challenged as it reinforces children and youth as passive rather than active in their health and well-being (Marjorie Montreuil \& Carnevale, 2016). Recently, the perspectives of children and youth are being recognized as valuable (M Montreuil, Thibeault, McHarg, \& Carnevale, 2018; Marjorie Montreuil, Saint-Laurent, \& Carnevale, 2017; Riley, 2004; Sixsmith, Gabhainn, Fleming, \& O'Higgins, 2007), and new research focusing on the health and well-being of children and youth are increasingly taking a child-centered approach to help capture their perspectives (Redmond, Skattebol, \& Saunders, 2013).

A major gap in literature on health of First Nations children and youth is their perspective concerning their health and well-being. Therefore, the aims of this research study were to understand how children and youth in Winneway, Quebec view health and well-being and to determine what they consider to be major health and well-being concerns. This research will help to expand knowledge of how children and youth learn, view, evaluate, and participate in their health. Exploring health and wellbeing from the perspective of children and youth may help to focus community health interventions as well as illuminate areas of health and well-being that are often overlooked. Additionally, given that 
school environments are a primary setting for promoting both well-being and culturally-attuned health education, this type of research can help inform interdisciplinary improvements in line with TRC Calls to Action that are essential for advancing culturally-sensitive practices.

\section{Terms}

As there is no clear separation between health and well-being, the terms will be used interchangeably as health/well-being. Additionally, there is no agreed upon age or definition for child and youth in the community, therefore the term child will be used to describe primary school students (kindergarten to grade 6) and youth will be used to describe secondary school students (grade 7 to grade 11).

\section{Methods}

The study used a focused ethnography methodology with a participatory approach, guided by an Indigenous decolonizing research framework. This framework required the study to include Indigenous members as part of the research team while seeking Indigenous guidance in the formation of the research question, how to approach the question, and how to build trust with the community (Bartlett, Iwasaki, Gottlieb, Hall, \& Mannell, 2007). This was achieved through collaboration with a community research partner (P.P.), as well as through nine Indigenous key informants. All key informants helped to develop interview questions and guided participant recruitment. Data were collected through semistructured interviews with key informants (KI) and child and youth participants, participant observations (PO), and analysis of key texts. KIs were expert sources of information who, based on their personal skill or position within the community, could provide deep insight into the culture and community (Marshall, 1996). All nine KIs in the study were Indigenous and eight were members of LPFN.

Purposeful non-random sampling was used to recruit child and youth participants. Participants were approached in person by the primary researcher (A.K.), and both consent from guardians and assent from children and youth were obtained. A total of fifteen interviews were conducted with 15 participants (10 females) ranging from grades one to eleven (ages six to seventeen); eight were primary school students (ages six to twelve) and seven were secondary school students (ages thirteen to seventeen). All interviews were conducted in English, audio-recorded, and transcribed verbatim. Interviews ranged from 18-53 minutes in duration and were conducted in private rooms. Participant initials have been changed to protect confidentiality and anonymity.

POs occurred in daily environments during a wide array of circumstances in order to make observations of culture through participants' actions (Venzon Cruz \& Higginbottom, 2013). POs with children are important because focused interviews with children may be new to them and may result in 
the study failing to fully capture the participant's experiences (Carnevale, MacDonald, BluebondLangne, \& McKeever, 2008). POs in this study were collected through many community events such as ceremonies and parties. The POs focused on activities, discussions, and interactions of children and youth at events. Key texts were identified through key informants. An example of a key text is the Declaration of the Rights of First Nations Children (Assembly of First Nations Quebec-Labrador, n.d.).

\section{Data Analysis and Rigour}

In accordance with ethnographic research methods, data were analyzed in four phases: domain analysis, taxonomic analysis, componential analysis, and thematic analysis (Polit \& Beck, 2008). Domain analysis identified broad categories from the raw interviews and included open codes, such as "No one teaches you about what it means to be healthy, it is something you feel". Taxonomic analysis grouped and coded the key themes from domain analysis. These codes were then compared with the data to establish analytical categories, such as "children describe health/well-being by how they feel". Componential analysis identified relationships among the taxonomic coding terms and examined them for similarities, differences, and subcategories. It was during this phase that the data were compared to ensure that no codes were overlapping. During the final phase, thematic analysis, the results reported in this paper were uncovered to project a holistic view and understanding of the research question and culture. To help ensure methodological rigour, three triangulation strategies were used: (1) interacting with multiple data sources (POs, interviews, and key texts); (2) a group activity with students that was designed to reflect and gain feedback on preliminary themes; and (3) data were independently reviewed and compared by authors A.K., F.C., and M.E.M.

\section{Relationship Community}

As part of a new collaboration between the community of Winneway, the Ingram School of Nursing, and the Views on Interdisciplinary Childhood Ethics Team (VOICE) at McGill University, Winneway became the focus of this study. The community of Winneway is in the region of AbitibiTémiscamingue in northwest Quebec and is home to the Long Point First Nation (LPFN). Its band of Algonquin people has 888 members, of which approximately 478 live in the community of Winneway. Data from Statistics Canada (2011) indicate that almost half of LPFN residents are under the age of 20, and the median age is 24.3 years. This study took place primarily at the Amo Ososwan School in Winneway, which opened in January 2017 and hosted 138 students ranging from pre-kindergarten to grade 11 during the 2017-18 school year. The school has a gymnasium, cafeteria, 12 classrooms, and a nursing office for healthcare providers. About half of the staff at the school are from LPFN, including the school Director, Principal, and Vice Principal. 


\section{Results}

\section{A: How the community views children and youth}

These results are based on POs, KI interviews, and analysis of key documents, and are complementary to the voices of children and youth, which are expressed in the following sections B and C.

Children and youth are involved and considered essential to the community of Winneway. Most community gatherings and events focus on attendance and participation of children and youth and multiple key informants expressed the view that it takes a community to raise a child. The Declaration of the Rights of First Nation Children by the Assembly of First Nations of Quebec and Labrador (n.d.) highlights family, extended family, and birth parents as imperative to the rights and well-being of children. It includes language, culture, identity, adequate food and housing, education, empowerment, and recreational activities as major rights of First Nation children, and these values are expressed clearly by community members and at community events in Winneway. These values were expressed verbally during community event meetings that discussed safety and inclusion of children and youth, physically during community events when children and youth presented hand-made crafts and songs, and symbolically through events that focused on the welcoming, entertaining, or celebrating children, youth and their achievements. An example of these community values was demonstrated through the naming of two centres in the community where people gather: The Elder Centre and The Youth Centre. Algonquin traditions were often the basis for ceremonies, such as including a drum circle, presence and use of cedar, and/or a speech from the Chief or council member. The community is also proud of its expanding Culture Week; a week spent in the nearby forest during early October where the community lives as their ancestors did: hunting, cooking, drumming, beading and making crafts together.

\section{B: How children and youth view health/well-being}

\section{Health/well-being is multidimensional}

Children and youth participants view health/well-being to include a person's mind, body, and emotions. They view actions and behaviours that make them feel good as healthy. Although some children and youth specifically tied the word "health" to physical acts, such as healthy eating habits or exercise, these same students also described emotional and mental aspects of health they consider important to well-being. For these participants, health/well-being included the social acts of respecting and being nice to others. A few youth discussed another aspect of well-being - social well-being - in relation to their time spent with friends. They value their free time with friends and consider it an important aspect of their health/well-being. 


\section{Children and youth take responsibility for making decisions about their health/well-being}

Children and youth of all ages identified themselves as having responsibility for their health/well-being. They emphasized that they make choices regarding their health/well-being and consider themselves fundamental in their health. The most common examples of this was with food and diet; despite children and youth in Winneway having easy access to unhealthy foods, they believed that eating healthy is a choice. Participants identified fruits, vegetables, and wild meat as foods that are healthy, whereas pop, chips, candy, and chocolate were considered unhealthy. Many participants had both healthy and unhealthy food at home, yet they highlighted that it is their responsibility to make healthy food decisions. The following student described how her friend chooses to eat healthy:

Participant NM: "[Friend's name] is the only one who eats healthy."

Interviewer: "And is that something that she chooses to do or is it something her mom and dad give her?"

Participant NM: "She makes it in her own mind."

Although children and youth equated feeling good with being healthy, they acknowledged that foods they enjoy such as chips, chocolate, pop, and candy do not make them feel healthy. Children took initiatives in their learning, including use of the Internet, reading posters at school, and asking trusted adults about healthy foods. Youth identified their initiative in seeking health/well-being information by use of the Internet and social media. Youth turned to the Internet when they had specific health/wellbeing questions, such as diabetes prevention, exercise motivation, and career inspiration. One youth used YouTube for exercise ideas and another followed a healthcare professional on Snapchat to develop aspirations about possible future careers.

\section{Families are major teachers about how to live a healthy life}

Children and youth expressed positive feelings about their families, including foster families. Parents and foster parents were frequently cited as important teachers of health/well-being. Parents and foster parents taught their children directly about health/well-being by providing knowledge about healthy/unhealthy foods, diabetes, the impacts of smoking, alcohol and drugs, and reminding children to brush their teeth. They taught children indirectly through rules, chores, by providing supportive and caring environments, and by paying attention and spending time with them. Siblings, grandparents, aunts, uncles, and cousins were also highlighted as important to the health/well-being of children and youth. One youth emphasized the connection of her family to her well-being:

Participant NE: "It makes me feel happier when we [family] are all together." 
Siblings were particularly referred to affectionately and participants described reciprocal teaching and learning about health/well-being with their siblings. Older siblings were often cited as teachers about healthy foods, exercise, learning to walk, and how to treat others. One participant described how she looks up to her sister and learned how to comfort friends by watching her sister comfort friends. Another child explained that her older siblings taught her to walk and now she is teaching her baby brother to walk and will eventually teach him to listen.

\section{Children and youth navigate social influences and personal beliefs to attain health/well-being}

Children and youth described how they see, learn, and judge health/well-being behaviours of their peers, older students, and adults in the community. They identified positive health/well-being behaviours that they observed, such as their grandparents exercising in the community or a friend teaching another to draw and were critical of the bad or unhealthy behaviours they saw. Participants disagreed with their peers who do not listen, respect others, or help their parents. They described how listening is positive for their well-being yet believe many students choose not to listen to teachers and parents. One participant described her pride:

Participant RR: "And my day is going great today and I'm starting to get good."

Interviewer: "Good at what?"

Participant RR: “At listening to my teacher".

Interviewer: "And what's helping you to listen to your teacher better? Is that something that you're choosing to do or learning to do?"

Participant RR: "It's about choosing and learning."

Participants of all ages described the positive and negative impacts of peers and friends on their health/well-being and how navigating these influences can be difficult. Youth considered friends to be important to their social well-being but found it difficult to navigate peer pressure. One youth highlighted the importance of friends watching 'each other's backs,' which contrasted with the difficulty of navigating peer pressure when the same friends pressure youth to participate in unwanted activities.

Youth revealed that the use of drugs and alcohol are visible to children and youth and that they believed this is harmful to their health/well-being. Youth unanimously agreed that the consumption of alcohol and use of pills are not good for their health/well-being but they had mixed beliefs about the use of cannabis. Youth described a community acceptance towards cigarettes and rejection of cannabis, yet many youth believed it should be the opposite. Youth participants believed that cigarettes are unhealthy, 
but that cannabis is not as detrimental to their lungs and, combined with its potentially therapeutic effects, does not have a negative impact on well-being. Youth related this positive effect of cannabis to the need to feel calm and relaxed. Although they sometimes used exercise and video games to reduce stress, most discussed cannabis as enjoyable and effective in reducing their stress.

Participants of all ages described teasing and bullying as common among their peers. They discussed negative impacts of teasing and bullying on the mental and emotional health/well-being of themselves and their peers; however, they had mixed opinions about the success of intervention by adults and teachers to address bullying. An elementary school participant described how she feels protected by her parents and teachers because they approach the child or their parent who was teasing her, but a high school student expressed a lack of confidence in teachers affecting bullying. Despite the presence of bullying, however, children and youth cared about and report intervening in the health/wellbeing of siblings, friends, and peers. Moreover, youth considered themselves role models for younger children and youth in the community. One participant described how they take cigarettes away from their younger sibling because smoking is bad for their sibling's health, and another described how he shares his food with classmates if they are hungry and without food. One youth emphasized the importance of role models:

Participant ZN: "Me, I'm just trying to make my own path cause I have no one to look up to. Just me myself and I... and my little brother. So, I always try to find my way and make it a good way for my little brother to pass through."

Youth acting as role models was also observed in PO sessions, such as in gym classes, where older students taught younger students how to play sports such as badminton and volleyball.

\section{Health/well-being are fostered and supported by the overall community}

The new school was considered by participants to be positive for their health/well-being. They believed that the school provided a safe and positive learning environment for children and youth and that teachers aimed to encourage and motivate students through recognizing their achievements. Children and youth cited psychologists, social workers, nurses, dentists, and hygienists as teachers and supporters of their health/well-being. Participants learned about healthy food and cavities from their dentist and dental hygienist, and trusted and sought out support from other healthcare professionals for their health/well-being needs. Children and youth found it difficult to discuss mental, social, and emotional well-being concerns but felt that psychologists and social workers supported them when they could articulate their concerns. One child demonstrated her trust in her social worker by explaining that she turns to her when she feels sad, and another youth highlighted how learning to talk about her feelings with her psychologist improved her happiness. 
Many participants felt that children and youth are valued by the community and the community has a responsibility in their health/well-being. It was mentioned by both key informants and youth that the community views children as the future generation and that to be a child in Winneway means to be part of the community. Participants reported feeling the community's responsibility in their health/wellbeing by workshops put on by the community and through a general sense of caring they feel from the community. One youth explained:

Participant NE: "Since we are such a small community everyone kind of takes care of each other."

However, one youth described a negative aspect of this community responsibility and involvement; they considered their actions to be watched and judged by the community and they found it difficult to redeem trust from the community.

Participant IN: "The last time someone trusted me was a long time ago. I did a mistake before doesn't mean I'm gonna do it again."

Youth highlighted traditional ceremonies as having continual positive impacts on their health/well-being. They looked to elders as leaders for traditional knowledge and ceremonies and considered their participation in traditional ceremonies to be supported by elders and the community.

\section{C: Significant health/well-being concerns of children and youth}

Four significant health/well-being concerns were identified by participants. These four concerns were poor eating choices that may lead to diabetes, difficulty expressing emotional and mental concerns, how children and youth treat others, and youth choosing to participate in unhealthy behaviours. Participants of all ages had access to unhealthy foods in Winneway, but participants believed that children and youth were choosing to eat unhealthy foods. This concern regarding poor eating habits was not only directed at peers but also at participants themselves. Children and youth learned about diabetes from their parents, grandparents, and the Internet, and knew that it could be prevented by eating fruits and vegetables over candy and pop. Despite this, they worried that they and their peers were at risk for diabetes because of poor dietary habits. The difficulty in expressing emotional and mental health concerns were described by participants of all ages; sources of these included bullying, grieving, and stress. Contrary to participants' trust in community psychologists and social workers, youth expressed fear that confidentiality may not be kept after sharing their concerns, which resulted in students 'keeping it in' and dealing with their issues on their own. Parents, social workers, and psychologists were all named as people that children and youth have turned to for help coping with mental and emotional concerns, although not all felt that talking helped them to handle or solve their problems. Compounding their concern of emotional and mental well-being was the health concern of teasing and bullying among 
elementary school students. Participants of all ages explained the negative impacts that teasing and bullying has on students' mental, social, and emotional well-being yet they all frequently observed and experienced teasing and bullying. One child talked about the difficulty that children face in addressing bullying in fear of being hurt again and how bullying is impacting how they choose to spend their free time:

Participant NM: "I stay inside and play videogames cause I don't want to get hurt outside sometimes."

Finally, youth in Winneway expressed a concern for themselves and their peers who were choosing to participate in unhealthy behaviours. Specifically, they felt that youth were not listening to teachers and were using drugs and alcohol. They believed that drugs in the form of pills and alcohol were beginning to affect the academic and athletic success of some and they were concerned that some youth will have difficulty carrying on the future generation. One youth described:

Participant $\mathrm{ZN}$ : "the people that will carry on this generation and keep it going on to the next one. But these days there is a lot of people getting into drugs. I don't think they will be able to carry it on. The healthy people, they will try to carry it on as long as they can."

The above results clearly demonstrate the engagement of children and youth toward their health/well-being but also highlight the involvement of parents, foster parents, families, and community as imperative to their health/well-being. While independence is displayed, learning comes from multiple sources and it is a combination of this that affects the health/well-being of children and youth.

\section{Limitations}

This is the first study to explore the views of Indigenous children and youth on their health/wellbeing; therefore, the transferability of the results to other Algonquin or Indigenous communities across Quebec and Canada is unknown. Although, only 15 children and youth participated in interviews and perspectives on health may be very individual, this study included the involvement and support by the whole community of Winneway and the unique perspectives of children and youth concerning their health/well-being reported in this study were widely held.

\section{Discussion}

The main results from this study highlight how children and youth in Winneway view their health/well-being as multidimensional, which includes physical, mental, emotional, and social aspects. Children and youth make decisions, navigate social influences, and personal beliefs to attain health/wellbeing, but they also have support from their family and community. The main health/well-being 
concerns, as expressed by children and youth, include poor eating choices that may lead to diabetes, difficulty expressing emotional and mental concerns, treatment of other children and youth and, for youth, participation in unhealthy behaviours. To our knowledge, this is the first research of its kind that explores Indigenous children and youth's understandings and views of health/well-being as well as their understanding of their health/well-being concerns in their community. The findings in this paper demonstrate the valuable perspective that children and youth have; in particular, their participation in their health/well-being, as well as their views on healthy eating and cannabis.

Participants of all ages described personal responsibility for a wide range of health/well-being decisions, but most commonly for food choices. Since children and youth have access to unhealthy foods, they displayed a strong sense of accountability toward food choices. They respected friends who they believed made healthy-eating choices, and expressed concern for friends and themselves who struggled to eat healthy. Obesity and food security with access to healthy foods are a major concern for Indigenous communities (Sherman, Macdonald, Carnevale, \& Vignola, 2011; Wakani, Macdonald, Carnevale, Bernier, \& Wawatie, 2014). However, our results illuminate an important aspect of this concern, which is the recognition, motivation, and choice that children and youth make regarding their diet.

Youth's beliefs that cannabis is not harmful and serves as a source of anxiety or stress release are important findings. A technical report published by the Canadian Centre on Substance Abuse (2013), which conducted a qualitative study into the use of cannabis among teenagers, reported similar positive views by some youth on the effects of cannabis. This demonstrates that youth in Winneway are not alone in their beliefs. With the legalization of cannabis by the federal Government of Canada in October 2018, considerations for how Canadian youth view cannabis are vital, especially as youth are likely to be targeted for cannabis sales. Although there are many negative health/well-being effects of cannabis that relate to the concerns of youth, such as its relation to school performance and achievement and physical health risks (Volkow, Baler, Compton, \& Weiss, 2014), Indigenous youth's views relating cannabis to health/well-being are unknown.

Current research is increasingly recognizing children and youth as active agents in their everyday health/well-being (Clavering E. K. \& McLaughlin J., 2010; Huang, O’Connor, Ke, \& Lee, 2016; Lees et al., 2017; Marjorie Montreuil \& Carnevale, 2018); however, this is still scarce in the Indigenous context. Our results demonstrate children and youth can make valuable contributions towards their health/wellbeing, and that they convey a strong sense of responsibility for their health/well-being. They highlight their involvement in their health /well-being through seeking information and navigating knowledge, feelings, and personal expectations, but they also express health/well-being concerns that stem from their choices. Two studies conducted in the neighbouring Algonquin community, Rapid Lake, focused on addressing the well-being of youth through a lens of self-respect and oral health among school aged 
children and (Kooiman et al., 2012; Naidu et al., 2014). Kooiman et al. (2012) highlighted the holistic approach that youth have towards self-respect, similar to the view that children and youth in Winneway hold regarding their health/well-being. Additionally, Naidu et al. (2014) suggested that both parents and children have a considerable amount of oral health and hygiene knowledge, but that knowledge itself did not motivate or improve oral hygiene practices. These results reinforce that interventions that target education alone may not be sufficient at improving health/well-being, and rather the views that children and youth hold must be integrated into health/well-being interventions.

Literature focusing on health needs and disparities of Canadian Indigenous children and youth's health/well-being highlight obesity, diabetes, mental health and addictions, and childhood dental caries as main concerns (Ames, Rawana, Gentile, \& Morgan, 2015; Canada, 2011; Galloway, Young, \& Egeland, 2010; Greenwood, Leeuw, \& Naomi, 2012), all of which, except dental caries, are highlighted by the children and youth in Winneway. Much of the current literature relates these concerns to Indigenous peoples' colonial past and social determinants of health (Greenwood et al., 2012; Leeuw, Greenwood, \& Cameron, 2010). Research also suggests culturally-attuned approaches are key to reduce or prevent these health concerns (Ritchie, Wabano, Russell, Enosse, \& Young, 2014; Thira, 2014; Yi, Landais, Kolahdooz, \& Sharma, 2015). Though culturally-attuned and participatory approaches are supported by the TRC Recommendations (2015), no research beyond this has exclusively focused on Indigenous child and youth agency to understand health/well-being concerns from their perspective.

Although the four major health/well-being concerns were supported by key informants and literature, the ability demonstrated by children and youth in Winneway to articulate their concerns for the health/well-being of their friends, peers, siblings, and themselves shows their capability to see and evaluate the health/well-being behaviours and actions in their community. This unique research not only reveals relevant health/well-being concerns for children and youth in Winneway, but it is also the first research of its kind to display Indigenous children and youth's insight and understanding in their own health/well-being. Although the combination of health/well-being concerns identified by participants are unique to this community, the ability of children and youth to express their understanding of these concerns is likely not unique to this community.

Future research in the community of Winneway should focus on exploring the ways in which children and youth maintain motivation and empowerment to make positive health/well-being choices, such as eating healthy, as well as the additional support they require to continue making positive choices. Research should also specifically explore mental and emotional well-being, and the beliefs regarding the use of cannabis among youth in Winneway and across Canada. Future research should aim to translate these perspectives into practice/interventions by health care professionals and educators to provide culturally-attuned service to this community. 


\section{Conclusion/s}

The findings in this paper highlight children and youth's view that health/well-being are multidimensional and are a combination of physical, mental, emotional, and social aspects. This study reflects the importance of family and community to the health/well-being of Indigenous children and youth and emphasizes the responsibility that children and youth have in their personal health/well-being. This study reinforces the valuable perspective that children and youth possess on their health/well-being, their participation and decision-making in their health/well-being, as well as their views on healthy eating and cannabis. Future research should explore the perspectives of children and youth from other First Nation, Inuit, and Métis communities across Quebec and Canada. Moreover, future health/wellbeing interventions targeting Indigenous children and youth should seek, incorporate, and respect their knowledge and perspective of the health/well-being concern. 


\section{References}

Ames, M. E., Rawana, J. S., Gentile, P., \& Morgan, A. S. (2015). The Protective Role of Optimism and Self-esteem on Depressive Symptom Pathways Among Canadian Aboriginal Youth. Journal of Youth and Adolescence, 44(1), 142-154. https://doi.org/10.1007/s10964-013-0016-4

Assembly of First Nations Quebec-Labrador. (n.d.). Declaration of the Rights of First Nations Children. Retrieved from https://www.cssspnql.com/docs/default-source/centre-de-documentation/affichedeclaration-droits-enfant-pn-rognee-eng.pdf?sfvrsn $=0$

Ball, J. (2004). As if Indigenous Knowledge and Communities Mattered: Transformative Education in First Nations Communities in Canada. The American Indian Quarterly, 28(3), 454-479. https://doi.org/10.1353/aiq.2004.0090

Bartlett, J. G., Iwasaki, Y., Gottlieb, B., Hall, D., \& Mannell, R. (2007). Framework for Aboriginalguided decolonizing research involving Métis and First Nations persons with diabetes. Social Science \& Medicine, 65(11), 2371-2382. https://doi.org/10.1016/j.socscimed.2007.06.011

Boksa, P., Joober, R., \& Kirmayer, L. J. (2015). Mental wellness in Canada's Aboriginal communities: striving toward reconciliation. Journal of Psychiatry \& Neuroscience: JPN, 40(6), 363. https://doi.org/10.1503/jpn.150309

Canada, H. (2011, September 26). Aboriginal Diabetes Initiative Program Framework 2010 - 2015. Retrieved April 3, 2018, from https://www.canada.ca/en/health-canada/services/first-nations-inuithealth/reports-publications/diseases-health-conditions/aboriginal-diabetes-initiative-programframework-2010-2015-health-canada-2011.html

Carnevale, F., MacDonald, M., Bluebond-Langne, M., \& McKeever, P. (2008). Using participant observation in pediatric health care settings: ethical challenges and solutions. Journal of Child Health Care: For Professionals Working with Children in the Hospital and Community, 12(1), $18-32$.

Clavering E. K., \& McLaughlin J. (2010). Children's participation in health research: from objects to agents? Child: Care, Health and Development, 36(5), 603-611. https://doi.org/10.1111/j.13652214.2010.01094.x

Crawford A. (2015). A National Suicide Prevention Strategy for Canadians--From Research to Policy and Practice. Canadian Journal of Psychiatry. Revue Canadienne de Psychiatrie, 60(6), 239-241. 
Galloway, T., Young, T. K., \& Egeland, G. M. (2010). Emerging obesity among preschool-aged Canadian Inuit children: results from the Nunavut Inuit Child Health Survey. International Journal of Circumpolar Health, 69(2), 151-157. https://doi.org/10.3402/ijch.v69i2.17437

Government of Canada. (2004, November 3). First Nations and Inuit Health [landing page]. Retrieved April 30, 2017, from http://www.hc-sc.gc.ca/fniah-spnia/diseases-maladies/index-eng.php

Government of Canada. (2016, September 15). Aboriginal Peoples in Canada: First Nations People, Métis and Inuit. Retrieved April 30, 2017, from http://www12.statcan.gc.ca/nhs-enm/2011/assa/99-011-x/99-011-x2011001-eng.cfm\#a6

Gracey M, \& King M. (2009). Indigenous health part 1: determinants and disease patterns. Lancet (London, England), 374(9683), 65-75.

Greenwood, M. L., Leeuw, D., \& Naomi, S. (2012). Social determinants of health and the future wellbeing of Aboriginal children in Canada. Paediatrics \& Child Health, 17(7), 381-384. https://doi.org/10.1093/pch/17.7.381

Huang, X., O’Connor, M., Ke, L.-S., \& Lee, S. (2016). Ethical and methodological issues in qualitative health research involving children: A systematic review. Nursing Ethics, 23(3), 339-356. https://doi.org/10.1177/0969733014564102

King, M., Smith, A., \& Gracey, M. (2009). Indigenous health part 2: the underlying causes of the health gap. Lancet (London, England), 374(9683), 76-85.

Kooiman, H., Macdonald, M. E., Carnevale, F., Pineda, C., Nottaway, W., \& Vignola, S. (2012). Minododazin: Translating an Algonquin Tradition of Respect into Youth Well-Being in Rapid Lake, Quebec. Pimatisiwin: A Journal of Aboriginal \& Indigenous Community Health, 10(1), 115.

Lees, A., Payler, J., Ballinger, C., Lawrence, P., Faust, S. N., \& Meads, G. (2017). Positioning Children's Voice in Clinical Trials Research: A New Model for Planning, Collaboration, and Reflection. Qualitative Health Research, 27(14), 2162-2176. https://doi.org/10.1177/1049732317726760

Leeuw, S. de, Greenwood, M., \& Cameron, E. (2010). Deviant Constructions: How Governments Preserve Colonial Narratives of Addictions and Poor Mental Health to Intervene into the Lives of Indigenous Children and Families in Canada. International Journal of Mental Health and Addiction, 8(2), 282-295. https://doi.org/10.1007/s11469-009-9225-1 
Montreuil, M, Thibeault, C., McHarg, L., \& Carnevale, F. (2018). Children's moral experiences of crisis management in a child mental health setting. International Journal of Mental Health Nursing.

Montreuil, Marjorie, \& Carnevale, F. A. (2016). A concept analysis of children's agency within the health literature. Journal of Child Health Care, 20(4), 503-511.

https://doi.org/10.1177/1367493515620914

Montreuil, Marjorie, \& Carnevale, F. A. (2018). Participatory Hermeneutic Ethnography: A Methodological Framework for Health Ethics Research With Children. Qualitative Health Research, 1049732318757489. https://doi.org/10.1177/1049732318757489

Montreuil, Marjorie, Saint-Laurent, O., \& Carnevale, F. A. (2017). The Moral Experiences of Children Living in Poverty: A Focused Ethnography. Journal of Childhood Studies, 42(2), 1-9.

Naidu, A., Macdonald, M. E., Carnevale, F. A., Nottaway, W., Thivierge, C., \& Vignola, S. (2014). Exploring oral health and hygiene practices in the Algonquin community of Rapid Lake, Quebec. Rural and Remote Health, 14(4), 2975.

Polit, D. F., \& Beck, C. T. (2008). Nursing Research: Generating and Assessing Evidence for Nursing Practice. Lippincott Williams \& Wilkins.

Public Health Agency of Canada. (2008, October 7). Aboriginal children: the healing power of cultural identity [education and awareness]. Retrieved April 19, 2018, from https://www.canada.ca/en/public-health/services/health-promotion/childhoodadolescence/programs-initiatives/aboriginal-head-start-urban-northern-communitiesahsunc/aboriginal-children-healing-power-cultural-identity.html

Redmond, G., Skattebol, J., \& Saunders, P. (2013). The Australian Child Wellbeing Project: Overview, 15.

Rice, K., Te Hiwi, B., Zwarenstein, M., Lavallee, B., Barre, D. E., \& Harris, S. B. (2016). Best Practices for the Prevention and Management of Diabetes and Obesity-Related Chronic Disease among Indigenous Peoples in Canada: A Review. JCJD Canadian Journal of Diabetes, 40(3), 216-225.

Riley, A. W. (2004). Evidence That School-Age Children Can Self-Report on Their Health. Ambulatory Pediatrics, 4(4), 371-376. https://doi.org/10.1367/A03-178R.1

Ritchie, S., Wabano, M., Russell, K., Enosse, L., \& Young, N. (2014). Promoting resilience and wellbeing through an outdoor intervention designed for Aboriginal adolescents. Rural and Remote 
Health Journal, 14. Retrieved from

http://www.rrh.org.au/articles/subviewnthamer.asp?ArticleID=2523

Sherman, J., Macdonald, M. E., Carnevale, F., \& Vignola, S. (2011). The Development and Implementation of a Type 2 Diabetes Prevention Program for Youth in the Algonquin Community of Rapid Lake, Quebec. Pimatisiwin: A Journal of Aboriginal \& Indigenous Community Health, 9(1), 219-244.

Sixsmith, J., Gabhainn, S. N., Fleming, C., \& O'Higgins, S. (2007). Childrens', parents' and teachers' perceptions of child wellbeing. Health Education, 107(6), 511-523.

https://doi.org/10.1108/09654280710827911

Thira, D. (2014). Aboriginal youth suicide prevention: a post-colonial community based approach. International Journal of Child, Youth and Family Studies, 5(1), 158-179. https://doi.org/10.18357/ijcyfs.thirad.512014

Venzon Cruz, E., \& Higginbottom, G. (2013). The use of focused ethnography in nursing research. Nurse Researcher, 20(4), 36-43.

Volkow, N. D., Baler, R. D., Compton, W. M., \& Weiss, S. R. B. (2014). Adverse Health Effects of Marijuana Use. New England Journal of Medicine, 370(23), 2219-2227. https://doi.org/10.1056/NEJMra1402309

Wakani, L., Macdonald, M. E., Carnevale, F., Bernier, I., \& Wawatie, J. (2014). Diabetes Bingo! Using participatory research to tackle diabetes with the Algonquin of Barriere Lake. Pimatisiwin: A Journal of Aboriginal, 11(3). Retrieved from https://mcgill.on.worldcat.org/atoztitles/link?genre=article\&isbn=\&issn=17057841\&title=Pimatisi win:\%20A\%20Journal\%20of\%20Aboriginal\%20\&\%20Indigenous\%20Community\%20Health\&v olume $=11 \&$ issue $=3 \&$ date $=20140101 \&$ atitle $=$ DIABETES $\% 20$ BINGO $\% 21 \% 20$ USING $\% 20$ PARTI CIPATORY\%20RESEARCH\%20TO\%20TACKLE\%20DIABETES\%20WITH\%20THE\%20AL GONQUIN\%20OF\%20BARRIERE\%20LAKE.\&aulast=Wakani\%2C\%20Laura\&spage=361\&pag es=361-376\& sid=EBSCO:Bibliography $\% 20$ of\%20Native $\% 20$ North\%20Americans\&pid=

Yi, K. J., Landais, E., Kolahdooz, F., \& Sharma, S. (2015). Factors Influencing the Health and Wellness of Urban Aboriginal Youths in Canada: Insights of In-Service Professionals, Care Providers, and Stakeholders. American Journal of Public Health, 105(5), 881-890. https://doi.org/10.2105/AJPH.2014.302481 